\title{
Flame-Flow Interactions and Flow Reversal
}

\author{
Gaurav Bansal ${ }^{\boldsymbol{\top} *}$, Hong G. $\operatorname{Im}^{\boldsymbol{\top} \dagger}$, John K. Bechtold ${ }^{\ddagger}$ \\ "Department of Mechanical Engineering, University of Michigan, Ann Arbor, MI 48109 \\ ${ }^{\ddagger}$ Department of Mathematical Sciences, New Jersey Institute of Technology, NJ 07102
}

\begin{abstract}
The interaction of a premixed methane/air flame with flow unsteadiness is studied computationally using a stagnation-point flow configuration. The problem is of fundamental interest and also relevant for turbulent combustion in the laminar flamelet regime. In the present study, of particular interest is the flame residing in a weakly strained flow field such that the flame is stabilized away from the viscous boundary layer adjacent to the stagnation plane and is free to move in response to flow perturbations. An unsteady sinusoidal strain rate field is imposed on the flame, and an extensive parametric study is conducted by varying the frequency and amplitude of strain rate fluctuation. It is found that for high frequencies and large amplitudes, flow direction reverses upstream of the flame, thereby establishing a new stagnation plane in the preheat zone ahead of the flame. This observation indicates that the flame strongly effects the upstream flow field and could also possibly explain the reported occurence of flow reversal in experimental studies of turbulent jet flames. Lewis number effects on flame-flow interaction and flow reversal is studied by investigating highly lean $(\mathrm{Le}<1)$ and rich $(\mathrm{Le}>1)$ hydrogen/air flames. Comparisons of the results are made with an earlier theoretical study of flame-flow interactions incorporating a hydrodynamic model [Z. Huang, J. K. Bechtold, M. Matalon, Combust. Theory Modelling 2 (1998) 115-133].
\end{abstract}

\section{Nomenclature}

$\mathrm{V}_{\text {inlet }}$ Flow velocity at nozzle inlet

A Amplitude of velocity fluctuation at nozzle inlet

$f \quad$ Frequency of velocity fluctuation at nozzle inlet

$\mathrm{V}_{0} \quad$ Initial velocity at nozzle inlet at time $=0 \mathrm{~ms}$

$\mathrm{S}_{\text {cons }} \quad$ Flame consumption speed (see Eq. 2)

$\mathrm{x}_{\text {stag }} \quad$ Stagnation plane position

$\mathrm{x}_{\text {flame }}$ Flame position (position of maximum heat release rate)

$\mathrm{V}_{\text {flame }}$ Unburned flow velocity at flame base

$\mathrm{S}_{\mathrm{d}}^{*} \quad$ Density weighted flame displacement speed

$\overline{\mathrm{x}_{\text {flame }}}$ Mean flame position in limit cycle

$\mathrm{x}_{\text {flame }}^{0}$ Initial flame position at time $=0 \mathrm{~ms}$

\section{Introduction}

The interaction of a premixed flame with a nonuniform flow field is an important subject in modeling turbulent combustion in the laminar flamelet regime, ${ }^{1}$ in which the characteristic flow and chemical time scales are such that flames retain their laminar structure, and the effects of turbulent eddies are represented by the wrinkling and straining of the flame front. Dynamics of premixed flames in a stagnation-point flow has been extensively studied as a canonical configuration to assess and quantify the flame behavior

${ }^{*}$ Corresponding Author, Email: gbansal@umich.edu, Present Affiliation: Postdoctoral Fellow, Center for Turbulence Research, Stanford University, Stanford, CA 94305

$\dagger$ Associate Professor, AIAA Associate Fellow

$\ddagger$ Professor 
in response to a well-defined strain rate parameter representing the fluid dynamic effects. A large number of experimental, ${ }^{2,3}$ numerical, ${ }^{4-6}$ and theoretical ${ }^{7-9}$ studies have used this configuration to examine the structure and extinction characteristics of premixed flames.

On the other hand, relatively few studies exist regarding the effects of flame on the flow field by means of the large gas expansion across the reaction zone. Most of the existing work on this issue focused on the events in the post-flame region in the context of flame-generated vortices, but the effects of flame on the upstream flow region have been largely neglected. In a recent experimental study, ${ }^{10}$ however, a reversal of the upstream gas velocity ahead of the flame was observed, suggesting that a significant level of modification in the upstream flow region by the flame can occur. From a practical standpoint, such a flow reversal may facilitate flame stabilization by transporting hot product gases to the upstream fresh reactant mixture. Such occurrence of combustion products upstream of the flame base has been observed experimentally. ${ }^{11}$ The effect also has a significant impact on turbulent premixed combustion modeling, as the flame-induced flow reversal may lead to a large variation in the local flame speed.

A possible explanation of the observed flow reversal may be found in the nonpremixed edge flame propagation phenomena, ${ }^{12-14}$ in which a reduction in the upstream flow velocity was found due to flow divergence around the curved edge flame. This mechanism was proposed as an explanation of the stabilization of the lifted turbulent flame base. However, the effect is limited by the density ratio, ${ }^{12}$ hence such a quasi-steady description is not sufficient to be responsible for the observed flow reversal phenomena.

To this end, Huang et al. ${ }^{15}$ investigated flame-flow interactions theoretically by considering a premixed flame stabilized in a stagnation-point flow. They employed a hydrodynamic model ${ }^{9}$ in which the flame was regarded as a surface of density discontinuity separating burned from unburned gases. The effects of a sinusoidally oscillating strain rate were examined, and reverse flow was observed for high frequencies of strain rate oscillation even at a moderate amplitude. The results therefore provide an important implication that significant flow field modifications can result from unsteady flame response.

Therefore, the objective of the present study is to computationally investigate the interaction of weakly strained premixed flames to unsteady fluctuations in strain rate, in order to identify the occurrence of the flow reversal in realistic flame conditions. Detailed chemistry and variable thermodynamic and transport properties are incorporated in the model. An extensive parametric study is conducted for methane/air flames to investigate the effects of frequency and amplitude of strain rate fluctuation. Results will be compared with the theoretical model of Huang et al. ${ }^{15}$ In addition, effects of Lewis number on the flame response and the extent of flow reversal will be investigated by studying very lean and very rich hydrogen/air flames. Implications of the findings in practical turbulent combustion systems are also discussed.

\section{Problem configuration and numerical method}

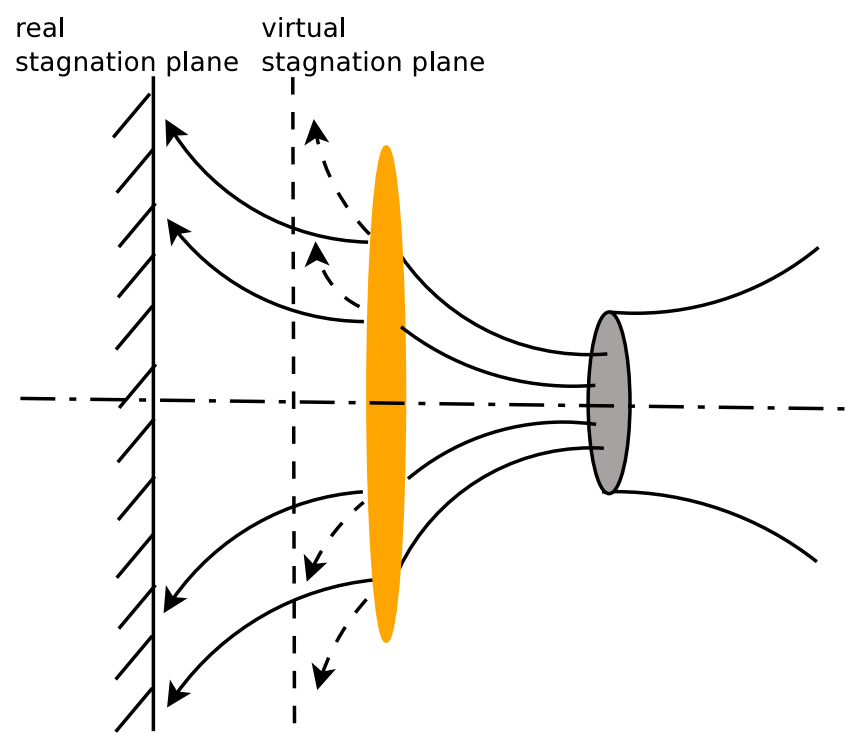

Figure 1. Model stagnation point flow configuration. 
The model configuration is an axisymmetric stagnation point flow as shown in Fig. 1. The figure schematically depicts the deflection of streamlines caused by the gas expansion as they pass through the flame; without gas expansion, the virtual streamlines and stagnation planes would be as shown by the dashed line. For this configuration, the unsteady one-dimensional conservation equations for mass, momentum, and energy, in symmetry coordinates, are derived along the centerline in a one-dimensional form. Details of formulation and numerical algorithm can be found in previous studies. ${ }^{16,17}$ The code is interfaced with CHEMKIN ${ }^{18}$ and Transport ${ }^{19}$ libraries for computing detailed reaction rates, and thermodynamic and transport properties. For the detailed reaction kinetics model, we adopted the GRI-mech $2.11^{20}$ for methane flames and the mechanism developed by Mueller et al. ${ }^{21}$ for hydrogen flames. Adiabatic boundary conditions are employed at the stagnation plane boundary. To impose unsteady strain rate on the flames, an unsteady velocity boundary condition of the form given in Eq. 1 is imposed at the nozzle inlet.

$$
\mathrm{V}_{\text {inlet }}(t)=\mathrm{V}_{0}(1-A(1-\cos (2 \pi f t)))
$$

where $A$ and $f$ correspond to the amplitude and frequency of the imposed sinusoidal velocity oscillation, respectively, and are the key parameters which will be varied to study the flame response. $\mathrm{V}_{0}$ is the initial velocity at time, $t,=0 \mathrm{~ms}$. To conduct unsteady simulations, a steady solution profile is first obtained for the nozzle inlet velocity of $\mathrm{V}_{0}$, and then the unsteady simulation is performed with the steady result as the initial solution.

The thermodynamic pressure of the system is fixed at $1 \mathrm{~atm}$, and the inlet temperature of the fuel/air mixture is fixed at $300 \mathrm{~K}$ for all the cases. The values of the fuel/air equivalence ratio and various unsteady parameters will be given in following sections. The size of the domain is fixed to $2 \mathrm{~cm}$ for all cases $(\mathrm{x}=$ 0 is the left stagnation plane boundary, and $\mathrm{x}=2 \mathrm{~cm}$ is the right nozzle inlet boundary). Flame location $\left(\mathrm{x}_{\text {flame }}\right)$ is defined as the location of maximum heat release rate within the domain. The consumption speed for hydrogen and methane flames is defined as: ${ }^{22}$

$$
\mathrm{S}_{\text {cons }}=\frac{\int \omega_{k} d \mathrm{x}}{\rho_{u}\left(Y_{k, b}-Y_{k, u}\right)}
$$

where $\omega$ is the reaction rate, $\rho_{u}$ is the upstream density, $Y$ is the mass fraction, subscripts $u$ and $b$ denote the unburned and burned sides, respectively, and $k$ corresponds to fuel species (hydrogen or methane).

\section{Flame response to flow unsteadiness}

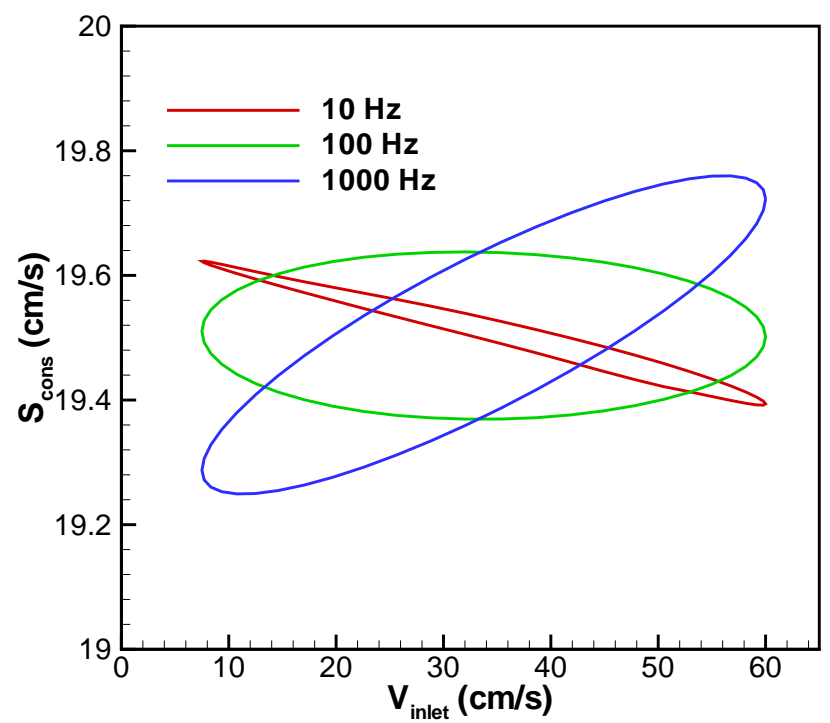

Figure 2. Consumption speed response to inlet velocity for different frequencies. $A$ is fixed to 0.4375 .

In this section, the results of flame response to unsteady strain rate are presented for the methane/air flame. Equivalence ratio of the methane/air mixture at the nozzle inlet is fixed at 0.7 for all cases, for which 


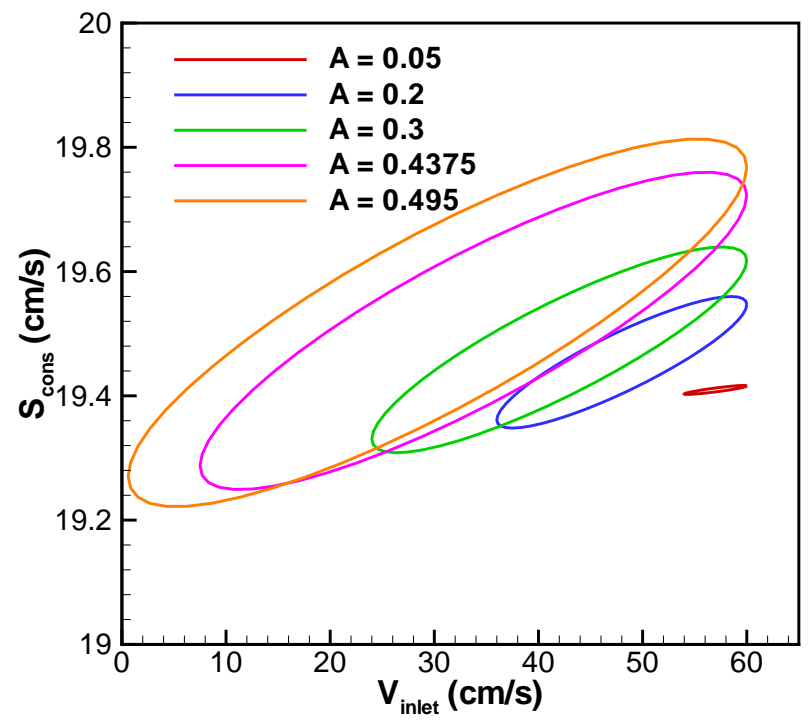

Figure 3. Consumption speed response to inlet velocity for different amplitudes. $f$ is fixed to $1000 \mathrm{~Hz}$.

a Lewis number of 0.97 is estimated at free-stream conditions. The initial velocity, $\mathrm{V}_{0}$, is fixed at $60 \mathrm{~cm} / \mathrm{s}$ for all cases. Two sets of parametric studies are performed. For first, the amplitude, $A$, of velocity fluctuation at nozzle inlet is fixed at 0.4375 , and frequency, $f$, of fluctuation is varied from 1 to $1000 \mathrm{~Hz}$. Thus the velocity at the nozzle inlet to vary sinusoidally from $60 \mathrm{~cm} / \mathrm{s}$ to $7.5 \mathrm{~cm} / \mathrm{s}$. For the second case, $f$ is fixed at $1000 \mathrm{~Hz}$ and $A$ is varied from 0.05 to 0.495 . Note that the maximum value of $A$ is 0.5 in order to ensure that flow velocity is directed towards the stagnation plane. Figures 2 and 3 show the phase diagram of the consumption speed versus the velocity at nozzle inlet $\left(\mathrm{V}_{\text {inlet }}\right)$ for the two cases. Due to the weak average strain rate and near-unity Lewis number, the consumption speed varies only slightly in both cases. The results show that, as expected, the flame response becomes attenuated as the amplitude is reduced. The phase lag is seen to vary a lot with frequency. As will be discussed later, the unsteady response of the flame speed and phase lag plays a role in the subsequent description of the flow reversal behavior.

Flow reversal is identified by the appearance of the stagnation point in the upstream region during a portion of each cycle (see Figure 7 of Huang et al. ${ }^{15}$ for an illustration of the flow field during the flow reversal.) Figure 4 shows the time evolution of flame position ( $\mathrm{x}_{\text {flame }}$, lines) and stagnation plane position $\left(\mathrm{x}_{\mathrm{stag}}\right.$, symbols) for different frequencies at a fixed amplitude of 0.4375 . For the computational configuration under consideration, the average flame position increases in time and reaches a limit cycle. During the evolution, the emergence of flow reversal is shown by the symbols. It should be noted that the stagnation plane position can only be identified when it lies upstream of the flame, and hence the symbols are only shown when the flow reversal actually occurs. For $f=1 \mathrm{~Hz}$, the flame is observed to flash back and does not obtain a limit cycle behavior. It is seen that the flow reversal behavior is more pronounced as the frequency increases. For $f=10 \mathrm{~Hz}$, flow reversal is only briefly observed during the transition period. A sustained real stagnation plane in the limit cycle is only observed for $f=100$ and $1000 \mathrm{~Hz}$. The inset of the figure shows the flame position and stagnation plane position in the limit cycle for $f=100 \mathrm{~Hz}$. It is observed that a phase lag exists between the flame position response and stagnation plane position response. Moreover, the flow reversal always occurs while the flame is moving upstream.

Figure 5 shows a similar plot for different amplitudes at a fixed frequency of $1000 \mathrm{~Hz}$. It is again observed that the average flame position moves upstream with time, with higher amplitude of velocity fluctuation causing the flame to sit closer to the nozzle. Despite the fact that higher $A$ causes the flame to reside closer to the nozzle, real stagnation plane is most prominently observed for higher amplitudes. All of these observed trends are consistent with the previous theoretical study. ${ }^{15}$

Figure 6 shows an instantaneous axial velocity and temperature profile for the flame for $f=1000 \mathrm{~Hz}$ and $A=0.48$, at time $=1.5 \mathrm{~ms}$, exhibiting a significantly wide region of positive velocity upstream of the flame. Considering that the nonreacting velocity profile would be a monotonic function from zero to $V_{\text {inlet }}$, the mechanism to create the reverse flow is the post-flame gas expansion combined with rapid withdrawal 


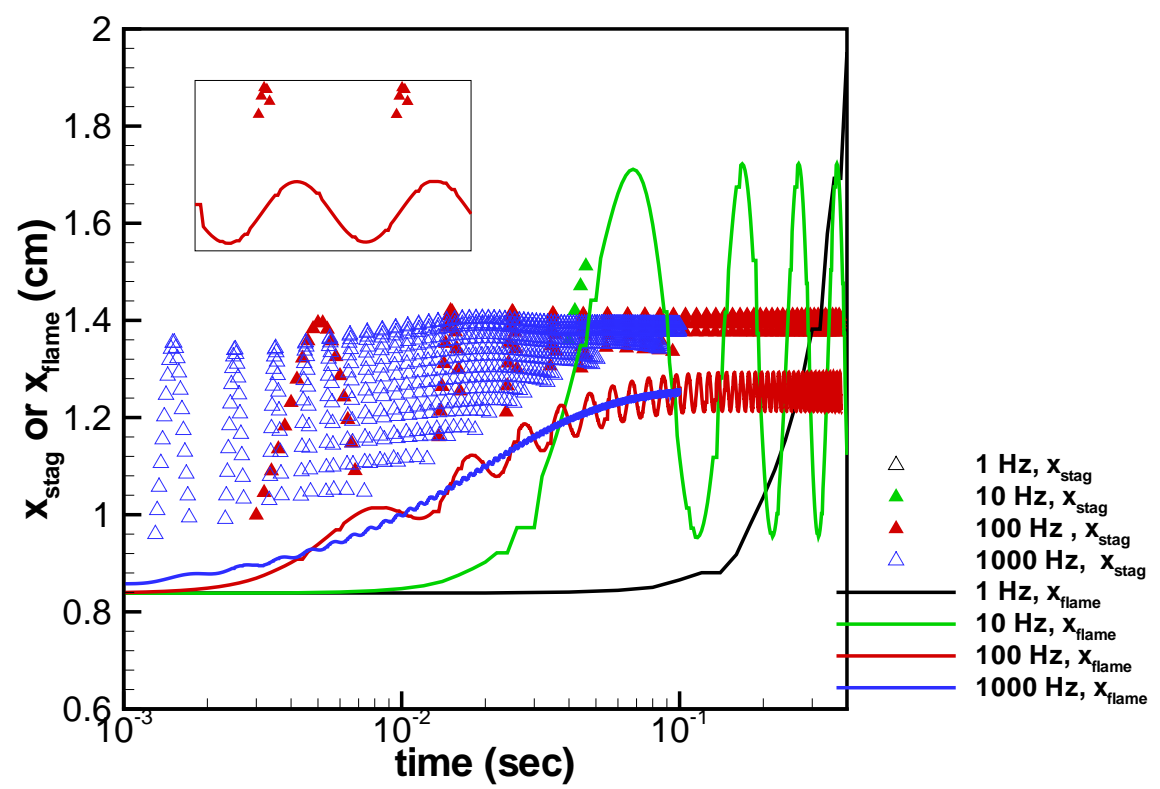

Figure 4. Flame position and stagnation plane position as a function of time for various frequencies, $A$ fixed to 0.4375 . Inset: Limit cycle response of flame position and stagnation plane position for $f=100 \mathrm{~Hz}$.

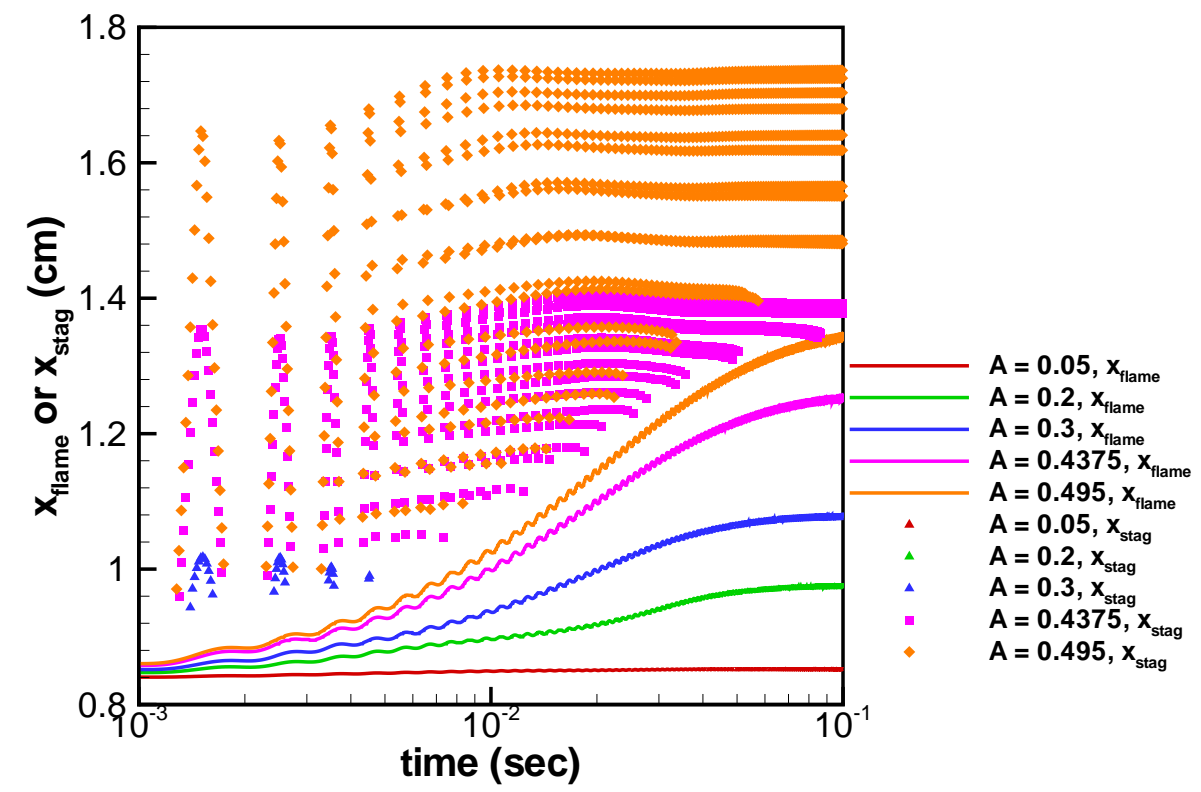

Figure 5. Flame position and stagnation plane position as a function of time for various amplitudes, $f$ fixed to 1000 Hz. 


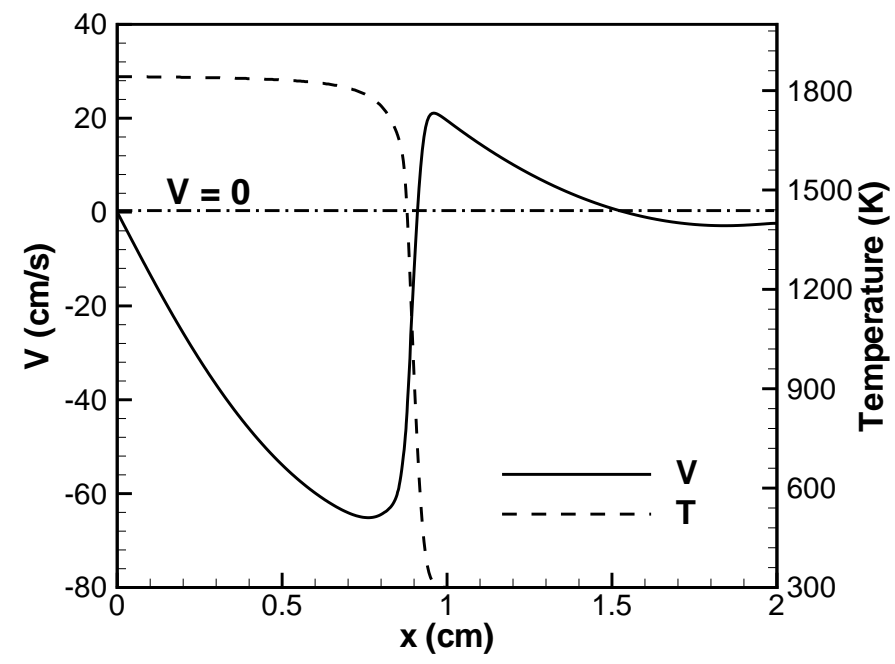

Figure 6. Flow velocity and temperature profiles in the domain at $t=1.5 \mathrm{~ms}$, for $A=0.48$ and $f=1000 \mathrm{~Hz}$.

of the upstream convective flow. It is important to note that this is a highly unsteady phenomenon, since such a condition cannot occur under a steady flow condition.

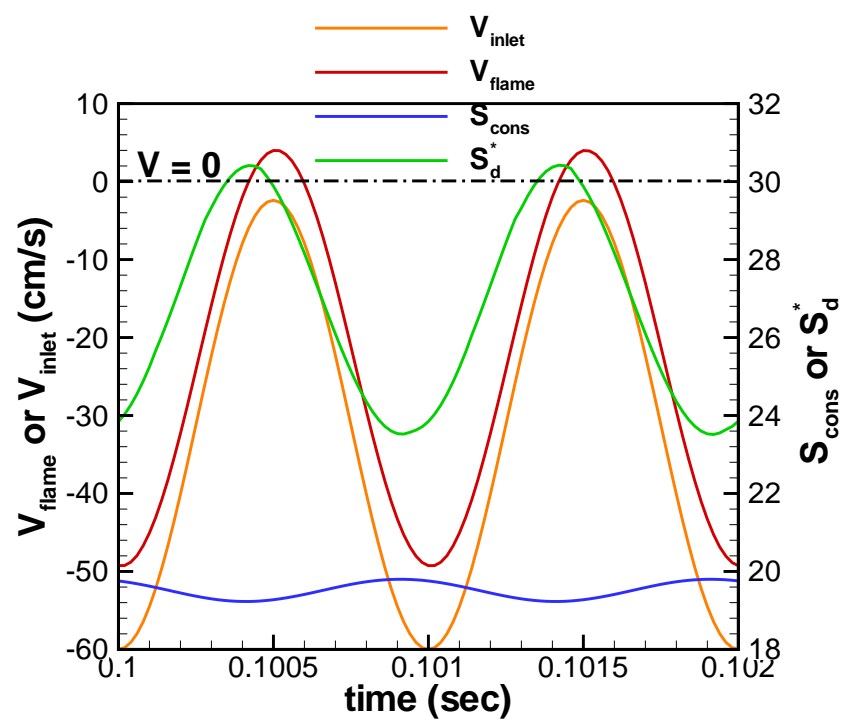

Figure 7. Important velocities as a function of time in the limit cycle for the condition shown in Fig. 6.

To further understand the complex flame-flow interaction behavior, Fig. 7 shows the temporal evolution of various important velocities in the limit cycle for the condition shown in Fig. 6. V Vlame is the unburned gas velocity computed at the flame base (the peak velocity ahead of the flame). $\mathrm{S}_{\mathrm{d}}^{*}$ is the density-weighted displacement speed relative to the local convective flow ${ }^{14}$ ), based on $\mathrm{H}_{2} \mathrm{O}$ species. $\mathrm{S}_{\mathrm{d}}^{*}$ is computed at the location where $Y_{\mathrm{H}_{2} \mathrm{O}}=0.03$, which is about $33 \%$ of the maximum downstream value of $Y_{\mathrm{H}_{2} \mathrm{O}} \approx 0.09$. Once again, the time duration during which $\mathrm{V}_{\text {flame }}$ becomes positive represents the duration of flow reversal upstream of flame. It is seen that $\mathrm{V}_{\text {flame }}$ and $\mathrm{V}_{\text {inlet }}$ are almost in phase, suggesting that there is no phase lag within the upstream convective zone. However, as mentioned in the previous paragraph, because of downstream gas expansion $V_{\text {flame }}$ is not just a mere translation of $V_{\text {inlet }}$. At this high frequency, the consumption speed $\left(\mathrm{S}_{\text {cons }}\right)$ remains almost unchanged despite the large velocity fluctuation. The displacement speed $\left(\mathrm{S}_{\mathrm{d}}^{*}\right)$, on the other hand, shows significantly large fluctuations. Moreover, there is a large phase lag 
between $\mathrm{S}_{\text {cons }}$ and $\mathrm{S}_{\mathrm{d}}^{*}$. This is not surprising considering that the definition of the displacement speed is based on the assumption of quasi-steady front propagation. This implies that the determination of the flame speed based on local front speed needs to be properly interpreted in characterizing highly unsteady flames.

Figure 7 clearly shows the mechanism primarily responsible for the flow reversal - it requires highly transient upstream convective flow withdrawal accompanied by a confined downstream gas expansion, as would occur in downstream-downstream flame interaction. This is precisely the condition encountered in the experiment in which a flow reversal was observed (see Figure 3 of Upatniekset al. ${ }^{10}$ ). In the following, additional effects contributing to the quantitative level of the flow reversal tendency are investigated by considering lean and rich hydrogen/air flames.

\section{Effects of preferential diffusion}

It is well known that the methane/air flames are diffusive-thermally neutral, hence the flame speed is rather insensitive to the unsteady strain rate fluctuations. As an important additional effect, we now consider the cases in which the flame speed depends strongly on the strain rate, and its impact on the flame-flow interaction behavior. This effect has not been considered in the previous theoretical study based on the hydrodynamic model. ${ }^{15}$

As a baseline reference, Figs. 8 and 9 show the flame temperature and consumption speed, respectively, as a function of $\mathrm{V}_{\text {inlet }}$ which is directly related to the strain rate. As expected, both quantities decrease with strain rate for the Le $>1$ case, and increase with strain rate for the Le $<1$ case. ${ }^{23}$ Consequently, flashback into the nozzle is more likely for the Le $>1$ flame, as will be reported in the subsequent results. Flashback was not observed for the Le $<1$ case since consumption speed reduces as inlet velocity is decreased. For unsteady calculations, as in the methane/air flame case, two parametric studies are conducted: one by varying frequency with $A$ fixed at 0.4375 , and the other by varying the amplitude with $f$ fixed at $1000 \mathrm{~Hz}$.

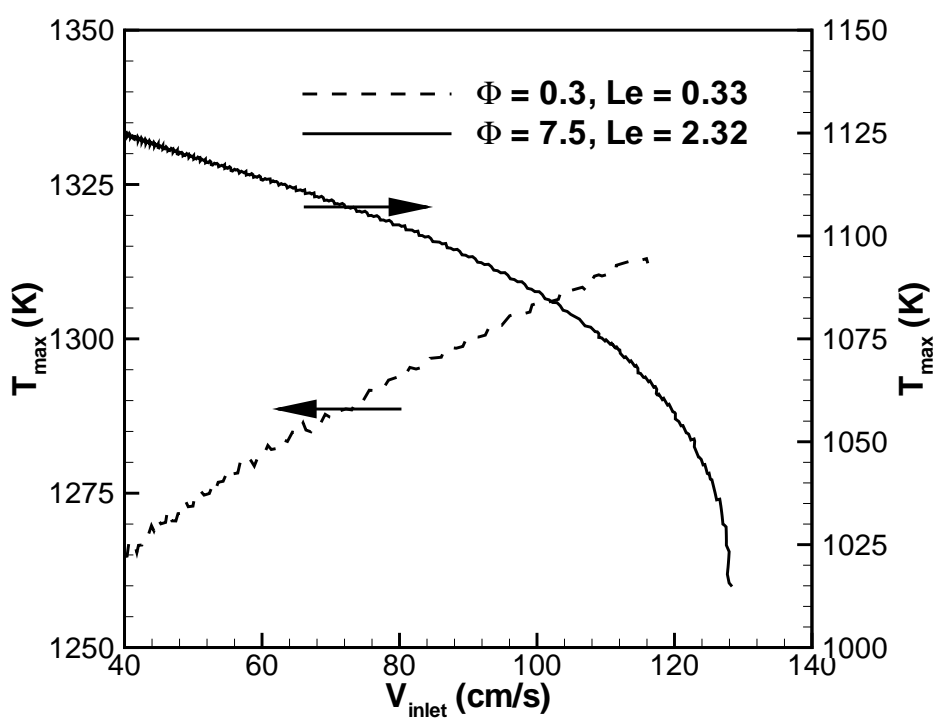

Figure 8. Flame temperature as a function of inlet velocity for rich and lean hydrogen flames.

Figures 10 and 12 show the flame position and stagnation plane position for different frequencies for the lean and rich cases, respectively. At such a large amplitude of the velocity oscillation, flashback is observed for the rich flame for $f=1 \mathrm{~Hz}$, as described earlier. Comparing the two, it is observed that the rich flame shows a higher sensitivity to the velocity oscillations, both in terms of the mean flame position in the limit cycle and its amplitude of oscillation. This is readily explained by the flame speed variation shown in Fig. 9; the rich flames further accelerates with the upstream velocity reduction, hence magnifying the amplitude of oscillation. In other words, the lean $(\mathrm{Le}<1)$ flame moves against the velocity swing, while the rich $(\mathrm{Le}>$ 1) flame moves with it. Considering the mechanism for flow reversal discussed in the previous section, this implies that the downstream gas expansion effect is further enforced for the lean flame, and attenuated for the rich flame. Consequently, the occurrence of the flow reversal is overall much more pronounced for the 


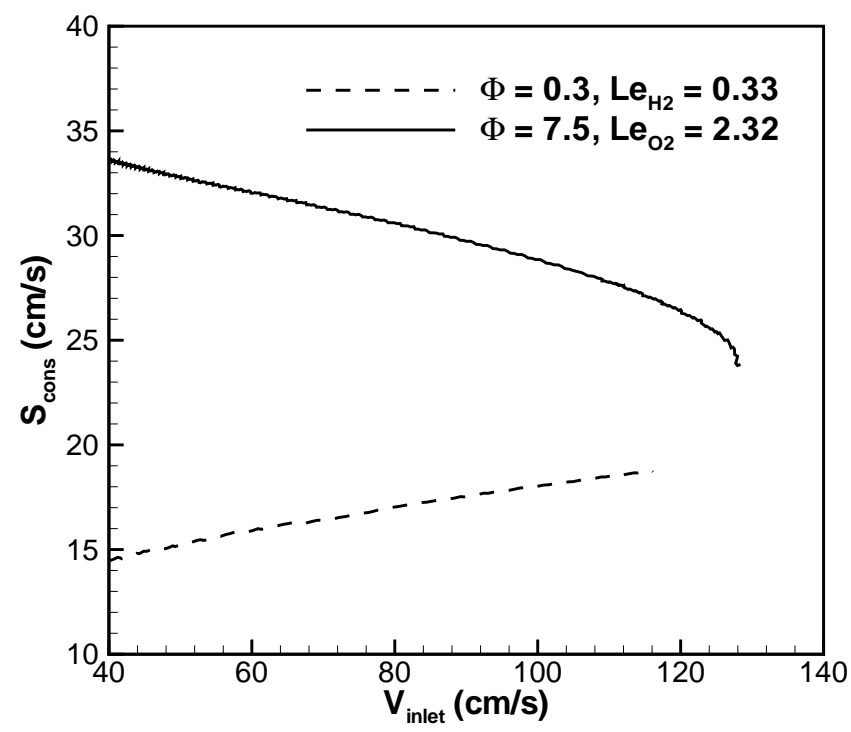

Figure 9. Consumption speed as a function of inlet velocity for rich and lean hydrogen flames.

lean flame. At high frequencies $(100$ and $1000 \mathrm{~Hz})$, some level of flow reversal is observed even for the rich flame because the flame response to the velocity fluctuation is highly attenuated.

Figures 11 and 13 show the amplitude dependence for the lean and rich flames, respectively. As explained earlier, at these high frequencies flow reversal is observed for both lean and rich flames as the flame speed response to the velocity oscillation is highly attenuated. In this case, the flow reversal tendency depends on the mean flame position; a faster flame is placed further away from the stagnation plane, hence attenuating the downstream expansion effect. Therefore, the fact that the rich flame is less likely to exhibit flow reversal in this case is directly related to the fact that the flame speed for the rich flame is higher.

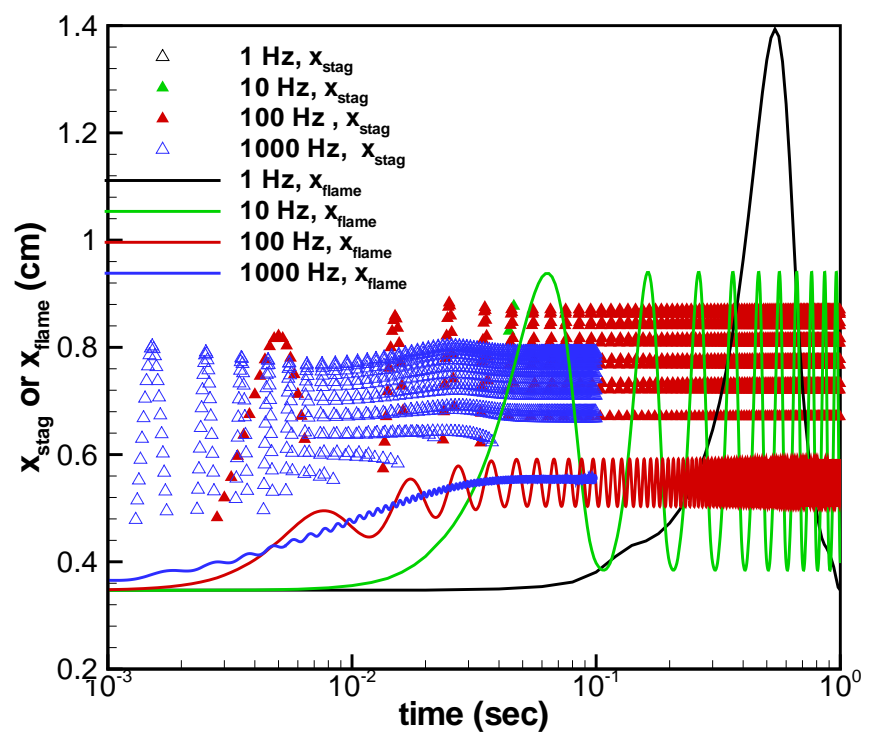

Figure 10. Flame position and stagnation plane position for the lean case as a function of time for various frequencies, $A$ fixed to 0.4375 .

However, considering the effect of velocity amplitude shown for the three cases (Figs. 5, 11, 13), it is consistently seen that flow reversal is more pronounced as the amplitude increases. This contradicts the earlier discussion in that, as the mean flame position moves further upstream, the upstream flow velocity (toward the flame) becomes higher and the downstream gas expansion effect becomes attenuated. To explain this behavior, Fig. 14 shows the mean flame position in the limit cycle $\left(\overline{x_{\text {flame }}}\right.$, lines$)$ and the maximum value 


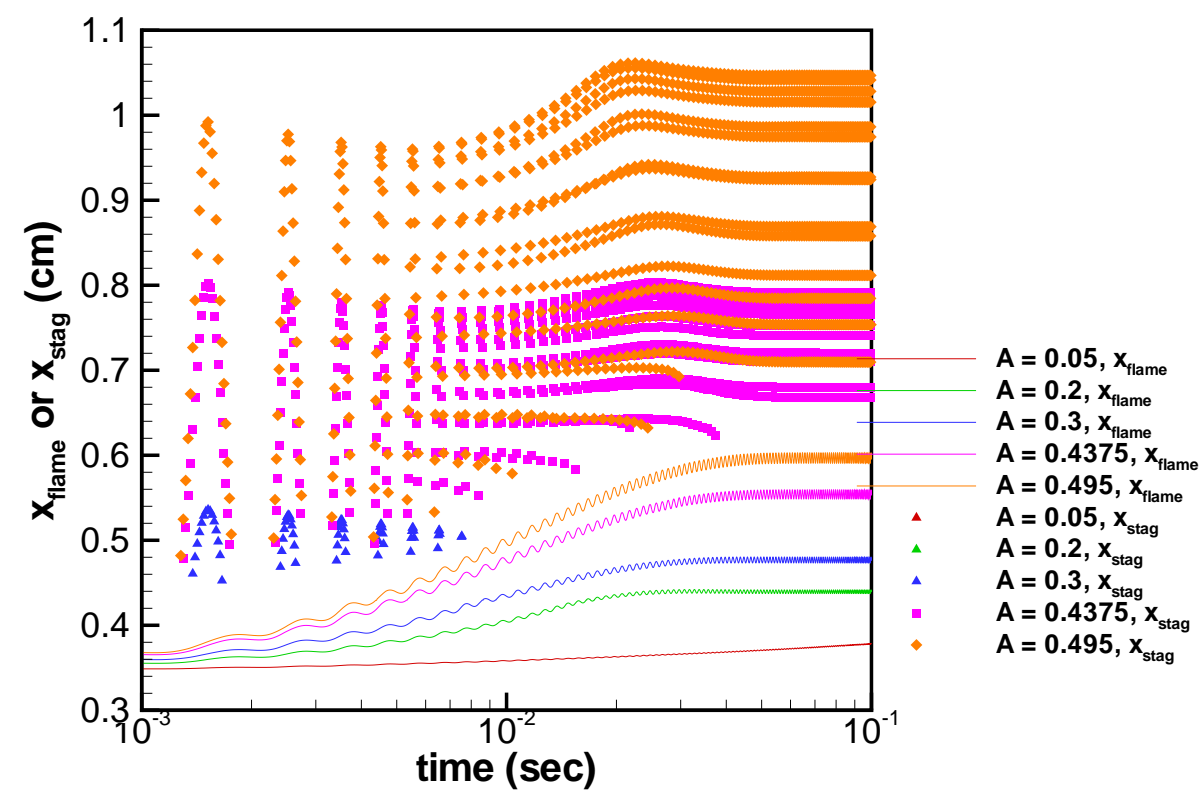

Figure 11. Flame position and stagnation plane position for the lean case as a function of time for various amplitudes, $f$ fixed to $1000 \mathrm{~Hz}$.

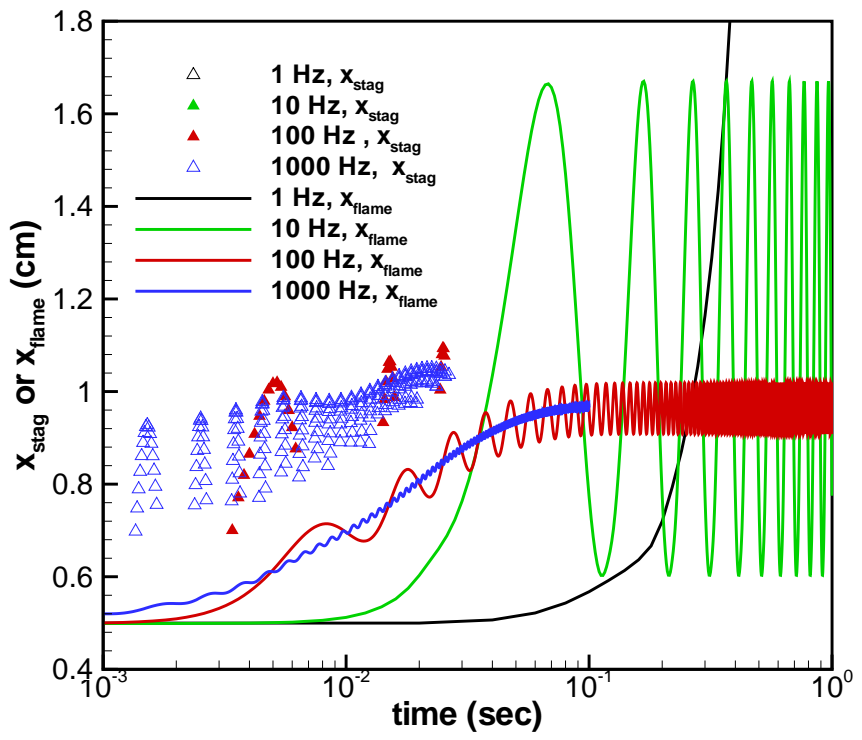

Figure 12. Flame position and stagnation plane position for the rich case as a function of time for various frequencies, $A$ fixed to 0.4375 . 


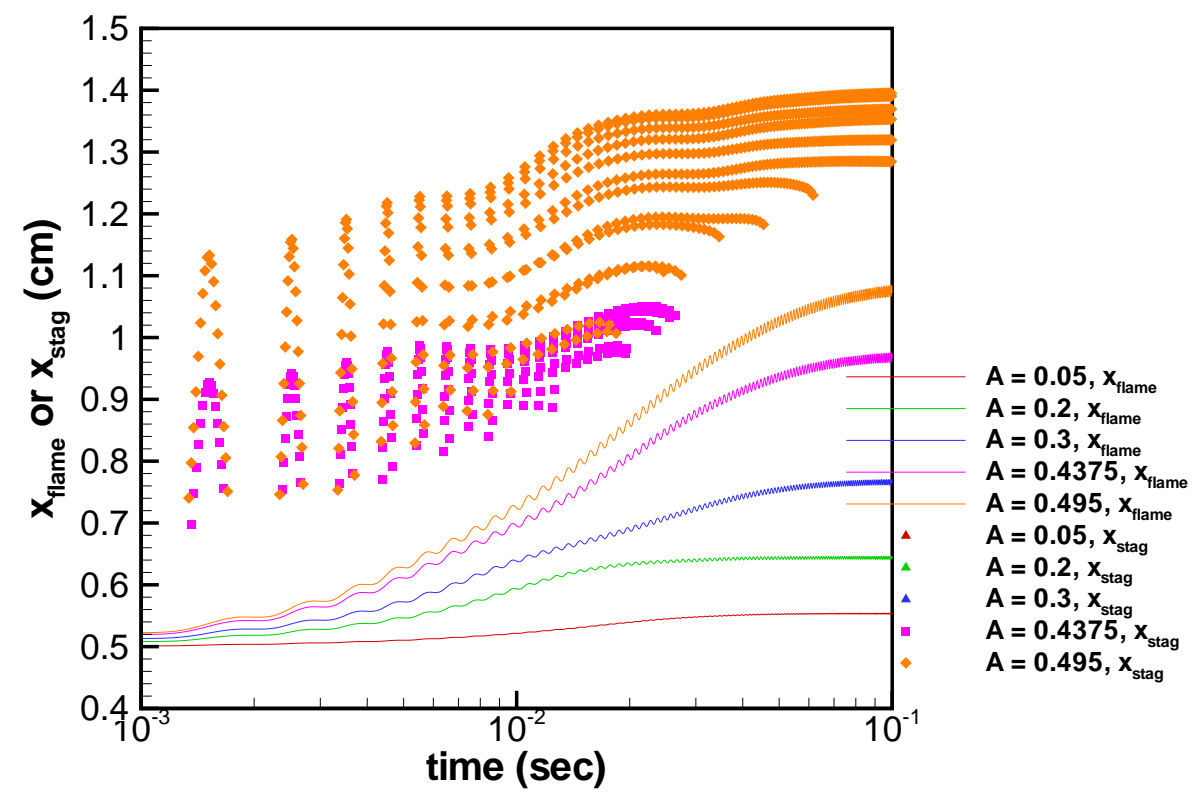

Figure 13. Flame position and stagnation plane position for the rich case as a function of time for various amplitudes, $f$ fixed to $1000 \mathrm{~Hz}$.

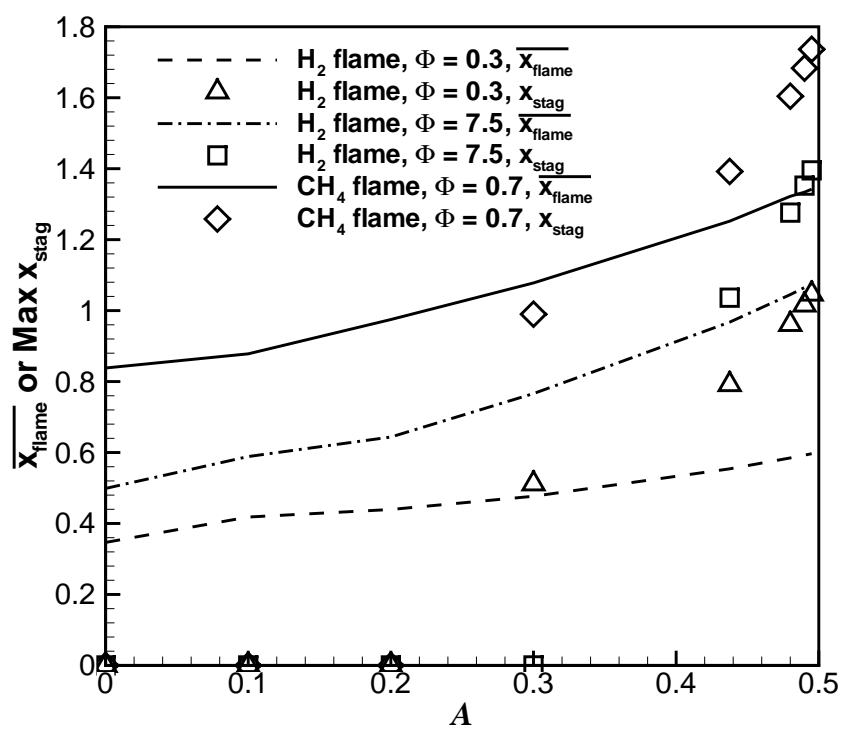

Figure 14. Mean flame position in the limit cycle and maximum value of stagnation plane position as a function of $A$, for the three flames. 


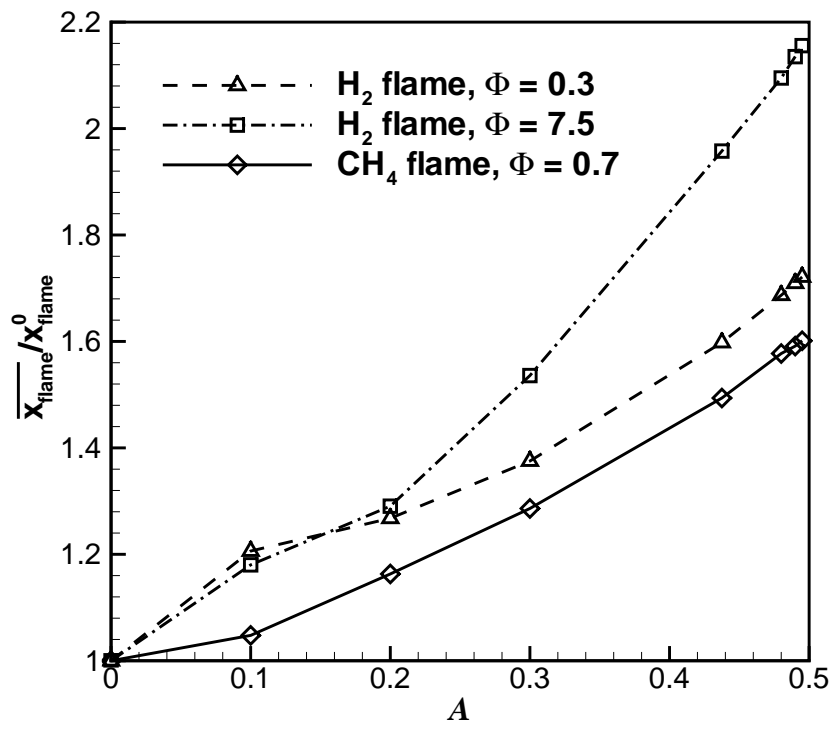

Figure 15. Mean flame position in the limit cycle normalized with flame position at initial time as a function of $A$, for the three flames.

of stagnation plane position ( $\mathrm{x}_{\text {stag }}$, symbols) as a function of $A$, for the three flames considered, at $f=1000$ Hz. As shown earlier, $\overline{\mathrm{x}_{\text {flame }}}$ increases monotonically and gradually with $A$. On the other hand, $\mathrm{x}_{\text {stag }}$ exhibits a more rapidly increasing trend. At the amplitude of approximately 0.3-0.4, the $\mathrm{x}_{\text {stag }}$ growth overtakes that of $\overline{\mathrm{x}_{\text {flame }}}$ hence the flow reversal emerges.

Figure 15 shows $\overline{\mathrm{x}_{\text {flame }}}$ normalized with the respective flame position at initial time. It is observed that the $\mathrm{CH}_{4}$ flame has the lowest value of normalized $\overline{\mathrm{x}_{\text {flame }}}$. This figure was motivated by the previous theoretical study, ${ }^{15}$ in which the mean flame position was found to increase to infinity at a critical value of $A$ between 0.4 and 0.6 for gas expansion ratio $\left(\sigma=\rho_{u} / \rho_{b}\right)$ lying between 4 and 6 . The approximate values of $\sigma$ (based on steady flame computation) for the present numerical study are $6.13,4.13$, and 3.49 for $\mathrm{CH}_{4}$, lean $\mathrm{H}_{2}$, and rich $\mathrm{H}_{2}$ flames, respectively. Despite the comparable $\sigma$ values, no critical value of $A$ is obtained in the present study at which the mean flame position goes to infinity. The reasons for this discrepancy may be attributed to the consideration of a finite domain in the present numerical study (as opposed to infinite domain assumption in the theoretical study), and also the presence of a diffusion layer in the present study which attenuates any disturbance imposed at the nozzle inlet (as opposed to considering the flame as a surface of density discontinuity in the theoretical model). Nevertheless, the present study captures the qualitative effects of the diffusive-thermal imbalance on the flow reversal trend through detailed computation - the effects that the hydrodynamic model could not account for.

\section{Practical relevance of flow reversal}

The observed flow reversal phenomena are relevant in many practical turbulent combustion systems. Some recent experimental results and their implications are highlighted in the following.

- Flow reversal at the base of a turbulent jet flame

In a cinema PIV study of Upatnieks et al., ${ }^{10}$ it was found that the instanteous gas velocities at the flame base and the flame velocities in a lifted turbulent jet flame are highly correlated, suggesting strong flame-flow interaction. Moreover, flow reversal was also observed at the base of a jet flame. Fig. 3 of Upatnieks et al. ${ }^{10}$ clearly shows the flow streamlines turning upstream ahead of the flame base, indicating reverse flow. The mechanism of flow reversal was explained by plotting the dilatation velocity field due to thermal expansion. It was observed that heat release causes the divergence of velocity vectors away from the base of the flame. It was conjectured that flow reversal may facilitate the flame stabilization process by forcing hot products upstream and could explain the reported occurrence of combustion products upstream of the flame base. ${ }^{11}$

Moreover, turbulent burning velocities are sometimes assumed to be equal to the average gas velocities 
in an equivalent non-reacting case at the corresponding fixed average position of the flame base. ${ }^{24}$ Reduced incident gas velocities at the flame base (due to flow reversal, and flame-flow interactions) would lead to average propagation velocities measured in reacting flows that are smaller than estimates based on the non-reacting case.

- Self-excited oscillations in combustors

Combustion instabilities occur in units as diverse as residential oil-fired heaters to blast furnaces, rockets, and jet aeroengines. Self-excited oscillations trigger combustion instabilities and are caused by the coupling between unsteady combustion and acoustic waves in the combustor. In the experimental study of Langhorne, ${ }^{25}$ large fluctuations of the flow velocity about the mean was observed due to the self-excited oscillations occuring in the combustor, causing flow reversal upstream of the flame base. In a related theoretical study on self-excited oscillations for a ducted flame by Dowling, ${ }^{26}$ flow reversal was predicted to occur during a part of the limit-cycle oscillation during which the flame moved upstream of the flame-holder. When flow reverses and the fluctuations in the velocity field are of the same order as the mean, the linear flame model does not hold, and must be extended to incorporate non-linear oscillations. The relationship between amplitude of oscillation of heat release and that of incoming flow velocity is shown to "saturate" when the flow velocity reverses, for finite-amplitude oscillations. These studies are for a confined flame in which acoustic pressure oscillations causes the fluctuations in incoming velocity and hence, flow reversal, whereas, the present study predicts strong variations in upstream flow velocity and flow reversal due mainly to thermal gas expansion across the flame. It is highly likely that both these processes might be simultaneously present in confined combustors, which may cause self-excited oscillations and hence affect the combustion dynamics of the system.

\section{Conclusions}

In the present study, the interaction of a premixed methane/air flame with flow unsteadiness was studied computationally using a stagnation point flow configuration. Of particular interest was a weakly stretched flame which sits away from the stagnation plane and is free to move. An unsteady fluctuation in strain rate was imposed by oscillating the flow velocity at nozzle inlet sinusoidally and the resulting flame-flow interaction is investigated. An extensive parametric study was conducted by varying the frequency $(f)$ and amplitude $(A)$ of inlet velocity fluctuation. Due to the weak mean strain, the variation in flame consumption speed is small even for large amplitude fluctuation in flow velocity. Presence of the flame, and hence thermal gas expansion, causes the flow streamlines to diverge as they pass through the flame front. A virtual stagnation plane (without gas expansion) may exist between the flame and the actual stagnation plane. Flow unsteadiness causes both the flame position and this virtual stagnation plane position to fluctuate, and for certain valus of $f$ and $A$, the virtual stagnation plane is observed to exist upstream of the flame and behaves as a real stagnation plane. This causes the flow to reverse direction upstream of the flame. The present computational study was intended to investigate this theoretical description based on a hydrodynamic model by incorporating realistic chemistry and transport.

Two parametric studies were conducted: (a) by varying $f$ and keeping $A$ fixed, and (b) by varying $A$ and keeping $f$ fixed. It was found that flow reversal becomes more pronounced at high values of $f$ and $A$. The primary physical mechanism for flow reversal is rapid upstream convective flow withdrawal accompanied by the downstream gas expansion in a confined region. It was also found that the conventional definition of the local flame displacement speed may lead to a large uncertainty for highly unsteady flame conditions.

Effects of lewis number on the flame-flow interaction and flow reversal were next investigated by studying lean $(\mathrm{Le}<1)$ and rich $(\mathrm{Le}>1)$ hydrogen flames, thereby incorporating the sensitivity of the flame speed to the velocity oscillation. For the lean case, the consumption speed increases as velocity increases, hence allows the flame to counteract against the upstream velocity fluctuation. On the other hand, the rich flame amplifies the oscillatory tendency and attenuates the downstream gas expansion effect. Consequently, the flow reversal was more pronounced for the lean hydrogen flames. At high frequencies, the flame response eventually attenuates, such that flow reversal was also observed for the rich flame. In the high frequency limit, the flow reversal tendency was then directly related to the mean flame position which is a function of the mean flame speed. For a given high frequency, the flow reversal was found to be more pronounced as the amplitude of the velocity fluctuation was increased, due to the higher sensitivity of the virtual stagnation plane fluctuation. 
Important practical implications of flow reversal were listed. Previous experimental studies of turbulent jet flames have shown the existence of flow reversal at the turbulent flame base. Flow reversal may force the hot product gases upstream and can possibly help in the flame stabilization process. Furthermore, care should be taken when determining turbulent burning velocities based on the upstream flow field measurement as the presence of the flame may significantly alter the upstream flow field, especially under highly unsteady conditions. Flow reversal may also occur when self-excited oscillations exist in confined combustors due to the coupling between unsteady combustion and acoustic waves. The present study provides an explanation that the presence of flow reversal in these devices may be the direct influence of post-flame gas expansion. These fundamental mechanisms of flow reversal need to be taken into account in proper description of the combustion dynamics.

\section{Acknowledgements}

During this study, GB and HGI were in part supported by the University Consortium on Low Temperature Combustion for High-Efficiency, Ultra-Low Emission Engines directed by the University of Michigan and funded by the Department of Energy. JKB was supported by the National Science Foundation grant DMS0807340 .

\section{References}

${ }^{1}$ N. Peters, Turbulent Combustion, Cambridge University Press (2000).

${ }^{2}$ C. K. Wu, C. K. Law, $20^{t h}$ Int. Symp. on Combustion (1985) 1941.

${ }^{3}$ J. M. C. Mendes-Lopes, H. Daneshyar, Combust. Flame 60 (1985) 29.

${ }^{4}$ V. Giovangigli, M. D. Smooke, J. Comput. Phys. 68 (1987) 327-345.

${ }^{5}$ G. Dixon-Lewis, $23^{\text {rd }}$ Int. Symp. on Combustion (1991) 305.

${ }^{6}$ R. Sankaran, H. G. Im, Proc. Combust. Inst. 29 (2002) 77-84.

${ }^{7}$ J. D. Buckmaster, $17^{\text {th }}$ Int. Symp. on Combustion (1979) 835.

${ }^{8}$ P. A. Libby, F. A. Williams, Combust. Flame 44 (1982) 287-303.

${ }^{9}$ E. Eteng, G. S. S. Ludford, M. Matalon, Phys. Fluids 29 (1986) 2172-2180.

${ }^{10}$ A. Upatnieks, J. F. Driscoll, S. L. Ceccio, Proc. Combust. Inst. 29 (2002) 1897-1903.

${ }^{11}$ M. M. Tacke, D. Geyer, E. P. Hassel, J. Janicka, Proc. Combust. Inst. 27 (1998) 1157.

${ }^{12}$ G. R. Ruetsch, L. Vervisch, A. Liñán, Phys. Fluids 7(6) (1995) 1447.

${ }^{13}$ L. Vervisch, Proc. Combust. Inst. 28 (2000) 11.

${ }^{14}$ H. G. Im, J. H. Chen, Combust. Flame 119 (1999) 436-454.

${ }^{15}$ Z. Huang, J. K. Bechtold, M. Matalon, Combust. Theory Modelling 2 (1998) 115-133.

${ }^{16}$ H. G. Im, L. L. Raja, R. J. Kee, L. R. Petzold, Combust. Sci. Technol. 158 (2000) 341-363.

${ }^{17}$ H. G. Im, L. L. Raja, R. J. Kee, A. E. Lutz, L.R. Petzold, OPUS: A Fortran Program for Unsteady Opposed-Flowed Flames, Report No. SAND2000-8211, Sandia National Laboratories (2000).

${ }^{18}$ R. J. Kee, F. M. Rupley, J. A. Miller, Chemkin-II, A Fortran Chemical Kinetics Package for the Analysis of Gas-Phase Chemical Kinetics, Report No. SAND89-8009B, Sandia National Laboratories (1991).

${ }^{19}$ R. J. Kee, G. Dixon-Lewis, J. Warnatz, M. E. Coltrin, J. A. Miller, A Fortran Computer Code Package for the Evaluation of Gas-Phase Multicomponent Transport Properties, Report No. SAND86-8246, Sandia National Laboratories (1986).

${ }^{20}$ C. T. Bowman, R. K. Hanson, D. F. Davidson, W. C. Gardiner, Jr., V. Lissianski, G. P. Smith, D. M. Golden, M. Frenklach, M. Goldenberg, http://www.me.berkeley.edu/gri_mech/

${ }^{21}$ M. A. Mueller, T. J. Kim, R. A. Yetter, F. L. Dryer, Int. J. Chem. Kinet. 31 (1999) 113-125.

${ }^{22}$ T. Poinsot, T. Echekki, G. Mungal, Combust. Sci. Technol. 81 (1992) 45-73.

${ }^{23}$ C. K. Law, C. J. Sung, Prog. Energy Combust. Sci. 26 (2000) 459-505.

${ }^{24}$ G. Kalghatgi, Combust. Sci. Technol. 41 (1984) 17.

${ }^{25}$ P. J. Langhorne, J. Fluid Mech. 193 (1988) 417-443.

${ }^{26}$ A. P. Dowling, J. Fluid Mech. 394 (1999) 51-72. 